Metallophysics and Advanced Technologies

Металофіз. новітні технол.

Metallofiz. Noveishie Tekhnol.

2019, vol. 41, No. 9, pp. 1127-1142

https://doi.org/10.15407/mfint.41.09.1127

Reprints available directly from the publisher
(C) 2019 G. V. Kurdyumov Institute for Metal Physics, National Academy of Sciences of Ukraine Published by license under

the G. V. Kurdyumov Institute for Metal PhysicsN.A.S. of Ukraine Publishers imprint. Printed in Ukraine.

\title{
ELECTRONIC STRUCTURE AND PROPERTIES
}

PACSnumbers: 61.50.Ks, 71.10.-w, 71.20.Be, 72.20.Dp, 72.20.My, 75.10.Dg, 75.30.Mb

\section{Фазовые диаграммы урана и его соединений. II. «Орбитальное стекло» (группы Галуа), магнитоэлектрические эффекты}

\author{
А. И. Мицек, В. Н. Пушкарь
}

Институт металлофизики им. Г. В. Курдюмова НАН Украины, бульв. Акаделика Вернадского, 36 , 03142 Киев, Украина

Порядок угловых моментов $\mathbf{J}_{\mathbf{r}}$ в металлическом уране может возникнуть путём упорядочения пар орбитальных моментов $\mathbf{L}_{\mathbf{r}}$ в форме «орбитального стекла». Представление многоэлектронных операторных спиноров и их Фурье-образов (флуктуаций химических (ковалентных) связей (ФХС)) даёт параметр «стекла» $P_{3}(T)=\left\langle\left(L_{r}^{z}\right)^{2}\right\rangle$ как функционал $\Phi \mathrm{XC}$ и функцию температуры $T$. Область металлического урана $\left(C_{3}\right)$ содержит $N_{3}$ элементов группы Галуа $\left(\Gamma \Gamma-\mathrm{G}_{3}\right)$ в виде пар узлов $(\mathbf{r}-\mathbf{R})$ иона $\mathrm{U}$ и их моментов $\left(\mathbf{L}_{\mathbf{r}} \uparrow \downarrow \mathbf{L}_{\mathbf{R}}\right)$. Ориентация $P_{3}$ вдоль $\mathrm{O} z$ при деформации $u_{33}$ определяет вклад магнитной восприимчивости $\chi_{z z} \sim P_{3}(T)$. Магнитоэлектрическое сопротивление $\Delta R_{33} \sim P_{3}(T)$. Ковалентная связь $6 d-6 d$ увеличивает температуру плавления на $\Delta T_{L} \sim 10^{2} \mathrm{~K}$ для $\mathrm{U}$ и $\mathrm{Cm}$ (имеющих по одному электрону $6 d$ ). Аномальный эффект Холла $R_{13}\left(P_{1}, P_{3}\right)$ обусловлен $\Phi Х \mathrm{X}$, как и остаточное электросопротивление $R_{0}$ переходных металлов, в частности $\mathrm{U}$.

Ключевые слова: порядок пар орбитальных моментов $\mathbf{L}_{\mathbf{r}}$ (группа Галуа, «орбитальное стекло»), магнитная восприимчивость, магнетосопротивление, эффект Холла.

Порядок кутових моментів $\mathbf{J}_{\mathbf{r}}$ в металічному урані може виникнути шляхом упорядкування пар орбітальних моментів $\mathbf{L}_{\mathrm{r}}$ у формі «орбітального

Corresponding author: Oleksandr Ivanovych Mitsek

E-mail: amitsek@gmail.com

G.V. Kurdyumov Institute for Metal Physics, N.A.S. of Ukraine, 36 Academician Vernadsky Blvd., UA-03142 Kyiv, Ukraine

Citation: O. I. Mitsek and V. M. Pushkar, Phase Diagrams of Uranium and Its Compounds. II. 'Orbital Glass' (Galois Groups), Magnetoelectrical Effects, Metallofiz. Noveishie Tekhnol., 41, No. 9: 1127-1142 (2019) (in Russian), DOI: 10.15407/mfint.41.09.1127. 
скла». Подання багатоелектронних операторних спінорів та їх Фурьєобразів (флуктуацій хімічних (ковалентних) зв'язків (ФХЗ)) дає параметр «скла» $P_{3}(T)=\left\langle\left(L_{r}^{z}\right)^{2}\right\rangle$ як функціонал $\Phi$ ХЗ і функцію температури $T$. Область металічного урану $\left(C_{3}\right)$ уміщує $N_{3}$ елементів групи Галуа $\left(\Gamma \Gamma-\mathrm{G}_{3}\right)$ у вигляді пари вузлів (r-R) іона U та їх моментів $\left(\mathbf{L}_{\mathbf{r}} \uparrow \downarrow \mathbf{L}_{\mathbf{R}}\right)$. Орієнтація $P_{3}$ вздовж $\mathrm{O} z$ при деформації $u_{33}$ визначає внесок магнітної сприйнятливості $\chi_{z z} \sim P_{3}(T)$. Магнітоелектричний опір $\Delta R_{33} \sim P_{3}(T)$. Ковалентний зв'язок $6 d-6 d$ збільшує температуру плавлення на $\Delta T_{L} \sim 10^{2} \mathrm{~K}$ для U і Cm (які мають по одному електрону $6 d)$. Аномальний ефект Холла $R_{13}\left(P_{1}, P_{3}\right)$ обумовлений $\Phi \mathrm{X}$, як і залишковий електроопір $R_{0}$ перехідних металів, зокремa U.

Ключові слова: порядок пар орбітальних моментів $\mathbf{L}_{\mathbf{r}}$ (група Галуа, «орбітальне скло»), магнітна сприйнятливість, магнітоопір, ефект Холла.

The order of angle moments $\mathbf{J}_{\mathbf{r}}$ in metallic uranium can be appeared due to ordering of pairs of orbital moments $\mathbf{L}_{\mathbf{r}}$ in 'orbital glass' form. Representation of the many-electron operator spinors (MEOS) and their Fourier images (chemical (covalent) bond fluctuations (CBF)) give 'glass' parameter $P_{3}(T)=$ $=\left\langle\left(L_{r}{ }^{z}\right)^{2}\right\rangle$ as CBF functional and function of temperature $T$. Metallic uranium region $\left(C_{3}\right)$ contains $N_{3}$ elements of Galois groups $\left(\mathrm{GG}-\mathrm{G}_{3}\right)$ in form of pairs of uranium ions sites $(\mathbf{r}-\mathbf{R})$ and their moments $\left(\mathbf{L}_{\mathbf{r}} \uparrow \downarrow \mathbf{L}_{\mathbf{R}}\right)$. Orientation of $P_{3}$ along $\mathrm{O} z$ under deformation $u_{33}$ determines the contribution of magnetic susceptibility $\chi_{z z} \sim P_{3}(T)$. Magnetoelectrical resistance $\Delta R_{33} \sim P_{3}(T)$. Covalent bond $6 d-6 d$ increases melting temperature by $\Delta T_{L} \sim 10^{2} \mathrm{~K}$ for $\mathrm{U}$ and $\mathrm{Cm}$ (which have one $6 d$ electron). Anomalous Hall effect $R_{13}\left(P_{1}, P_{3}\right)$ is caused by CBF as well as the residual electrical resistance $R_{0}$ of transition metals, in particular $\mathrm{U}$.

Key words: order of pairs of orbital moments $\mathbf{L}_{\mathbf{r}}$ (Galois group, 'orbital glass'), magnetic susceptibility, magnetoresistance, Hall effect.

(Получено 26 февраля 2019 г.)

\section{1. ЛОКАЛЬНЫЕ ОРБИТАЛЬНЫЕ МОМЕНТЫ В КВАНТОВОЙ СТАТИСТИКЕ}

Крайняя группа (5f) таблицы Менделеева выдает несколько сюрпризов. Первый - радиоактивные изотопы. На Земле их концентрация довольно мала. Второй - наличие локального орбитального момента $\mathbf{L}_{\mathbf{r}}$ при занулении спина $\mathbf{S}_{\mathbf{r}}=\mathbf{0}$ и углового момента $\mathbf{J}_{\mathbf{r}}=\mathbf{0}$. При этом, в отличие от группы $4 f$, между $\mathbf{L}_{\mathbf{r}} 5 f$ элементов возможно упорядочение типа «спинового стекла». Для его выявления воспользуемся топологией и квантовой статистикой.

Квантовая теория металлических актинидов (U, ...) строится на основе многоэлектронных операторных спиноров (МЭОС) в пространстве узлов $\mathbf{r}$, в частности в его части $C_{3}$, подчиняющейся группе Галуа $\left(\Gamma \Gamma-\mathrm{G}_{3}\right)$. Переход в импульсное $\mathbf{k}$-пространство рождает 
флуктуации химических (ковалентных) связей (ФХС). ФХС задает термодинамику металла U [1]. По аналогии с Се [2] объясняем зануление $\mathrm{S}_{\mathrm{r}}=0$ гибридизацией $6 f-6 d$ - и $5 f-7 s$-оболочек иона U. Отдельные $\mathbf{L}_{\mathbf{r}}$-моменты ионов $\mathbf{r}$ не создают группу Галуа (ГГ) и магнитный порядок.

Однако пары $(\mathbf{r}-\mathbf{R})$ образуют $\left(L_{r}^{i}, L_{R}^{i}\right)$ элементы группы Галуа $\left(\Gamma \Gamma-\mathrm{G}_{3}\right)$ в $C_{3}$-пространстве, при их ориентации вдоль оси $x_{i}=\{x, y, z\}$ кубического металлического урана.

Аналогично вводим группы $\left(\mathrm{G}_{1}, \mathrm{G}_{2}\right) \in C_{3}$.

При доминировании части $C_{3}>C_{1,2}$ получаем «орбитальное стекло» с параметром

$$
P_{3}=\left\langle L_{r}^{3} L_{R}^{3}\right\rangle \cong\left\langle\left(L_{r}^{3}\right)^{2}\right\rangle=P_{3}(T) .
$$

Он получается варьированием термодинамического потенциала (ТДП) как функционала $\mathbf{L}_{\mathbf{r}}$. В отсутствие спина и локального момента $\left(\mathbf{S}_{\mathbf{r}}=\mathbf{J}_{\mathbf{r}}=0\right.$ ) нечетные (по Акулову) эффекты (намагниченность $M_{s}$, эффект Холла) выражаются линейно через магнитное поле В [3]. Четные эффекты (магнитоупругость $\Lambda$, магнетосопротивление $\Delta R_{j j}$, аномальный эффект Холла $R_{13}$ ) выражаются через $P_{3}(T)$, см. ниже, как свойства «орбитального стекла». Для их расчета в представлении МЭОС используем полученный в [1] спектр ФХС ( $E_{\mathbf{k}}$ в kпространстве) как Бозе-частиц в пространстве Фока. Анизотропная локализация пар $\mathbf{L}_{\mathbf{r}}$-моментов направляется локализованной в пространстве $C_{3}$ деформацией $u_{33}$.

Используем аналогию с созданием ферромагнитной анизотропии (ФМА) как следствия $u_{j j}$ [4]. «Орбитальное стекло» $\left(\mathrm{P}_{3}\right)$ наводится магнитной частью ковалентной связи $\Gamma(\mathbf{r}-\mathbf{R})$. Ее градиенты (как $\left.\nabla r_{u}\right)$ связывают орбитальные факторы МЭОС $v_{L}\left(\mathbf{L}_{\mathbf{r}}\right)[1,2]$. Велика роль $u_{33}$ для выделения $\Gamma \Gamma-\mathrm{G}_{3}$, а также для четных эффектов, через магнитоупругость.

Топологическая классификация МЭОС и ФХС и стабилизация «орбитального стекла» анализируются в разд. 2, т.е. параметр $P_{3}(T)$. Вклад «орбитального стекла» в магнитную восприимчивость $\chi_{33}(T)$ учтен в разд. 3. Магнитоупругие параметры и т.п. рассчитываются как функция $P_{3}(T)$ и концентрации $N_{3}$ элементов $\Gamma \Gamma-\mathrm{G}_{3}$. Спектры коллективных возбуждений ФХС для $5 f$ - и $6 d$-зон рассчитаны в разд. 5 и применяются для расчета электросопротивления (ЭС) $R(T)$ переходного металла, в частности металлического урана. Выделяется (разд. 6 ) остаточное ЭС $R_{0}(0)$, зависящее от связи $\gamma$ между ФХС и зонными фермионами. Анизотропия спектров зонных фермионов (поверхности Ферми, эффективной массы $m_{i j}^{*}$, тензора релаксации $\left.\tau_{i j}\right)$, а также параметр аномального эффекта Холла $\left(R_{i j}\right)$ в пространстве $\mathrm{C}_{3}\left(\Gamma \Gamma-\mathrm{G}_{3}\right)$ выражаются через $N_{3}$ и $P_{3}(T)$ (разд. 7 и 9$)$. Увеличение температуры плавления U за счет $6 d-6 d$-связей рас- 
считано в разд. 8. Заключение и выводы даны в разд. 10 и 11.

\section{2. «ОРБИТАЛЬНОЕ СТЕКЛО». ГРУППЫ ГАЛУА (ГГ) В ПРОСТРАНСТВЕ $C_{j}$ ПАР ИОНОВ УРАНА}

Коллективизация отдельных орбитальных моментов $\mathbf{L}_{\mathbf{r}}$ невозможна из-за отсутствия прямой (обменной) связи. Ковалентная связь $\left(\mathbf{L}_{\mathbf{r}}-\right.$ $\mathbf{L}_{\mathbf{R}}$ ) здесь косвенная. Для ее выявления вводим аналогично [1] MЭОС $F_{\mathrm{r}}^{1}$ и $F_{\mathrm{r}}^{2} 5 f$-подоболочек

$$
\psi_{\mathbf{r}}^{+}=\xi_{1} F_{\mathbf{r}}^{1}+\xi_{2} F_{\mathbf{r}}^{2}+\xi_{d} D_{\mathbf{r}}+\sum_{\sigma} \xi_{\sigma} f_{\mathrm{r \sigma}}^{+}, \sum_{j} \xi_{j}^{2}=1 .
$$

Волновая функция - комбинация $5 f\left(F_{\mathrm{r}}^{n}\right), 6 d\left(D_{\mathrm{r}}\right)$ и $7 s\left(f_{\mathrm{r}}\right)$ зон. Металлический уран коллективизирует $7 s$-электроны в зонные фермионы. МЭОС при $\mathbf{S}_{\mathbf{r}}=0$ ( $n_{F}-$ число $5 f$-электронов)

$$
F_{\mathrm{r}}^{n}=\left\{F_{\mathrm{rL}}^{n}, v_{\mathrm{rL}}\right\}, v_{\mathrm{rL}}^{2}=\left(1+\mathbf{l L}_{\mathrm{r}}\right) /\left(2 n_{F}+1\right) .
$$

Орбитальные моменты содержит $F^{2}$-подоболочка (состояния $l=0$ и $\pm 1)$, для $e_{g}$-подоболочки $L=0$.

Условия локальности

$$
F_{\mathrm{rL}}^{n} \bar{F}_{\mathrm{rL}}^{n}=1,\left[F_{\mathrm{rL}}^{2}, \bar{F}_{\mathrm{RL}}^{2}\right]=\delta_{\mathrm{rL}} \delta_{\mathrm{LL}^{\prime}},\left[F_{\mathrm{k}}^{2}, \bar{F}_{\mathrm{q}}^{2}\right]=\delta_{\mathrm{kq}} /\left(N n_{F^{2}}\right)
$$

при переходе в $\mathbf{k}$-пространство

$$
F_{\mathbf{r}}=F_{0}+\sum_{\mathbf{k}} F_{\mathbf{k}} \exp (i \mathbf{k r}), \quad F_{\mathbf{k}}=\sum_{\mathbf{r}} F_{\mathbf{r}} \exp (-i \mathbf{k r} \mathbf{r})
$$

позволяют ввести ковалентную связь $(5 f, 6 d)-(6 d, 5 f)$

$$
H^{\mathrm{cov}}=-\sum_{\mathbf{r R}} \Gamma(\rho) F_{\mathrm{r}} D_{\mathbf{R}} \bar{D}_{\mathbf{r}} \bar{F}_{\mathbf{R}}, \quad \rho=\mathbf{r}-\mathbf{R},
$$

в k-представлении

$$
H^{\mathrm{cov}} / N=-\left\{\Gamma(0) F_{0} D_{0} \bar{D}_{0} \bar{F}_{0}+\sum_{\mathbf{k}} \Gamma(\mathbf{k}) F_{0} D_{\mathbf{k}} \bar{D}_{\mathbf{k}} F_{0}+\ldots\right\},
$$

где

$$
\Gamma(\mathbf{k})=\sum_{\vec{\rho}} \Gamma(\rho) \exp (i \mathbf{k} \rho), \Gamma(0)=\Gamma_{0} \xi_{2}^{2} \xi_{d}^{2}
$$

содержит в (2.6) анизотропную часть. Она, по аналогии с [4], появляется в пространстве $C_{3}$ группы $\left(\Gamma \Gamma-\mathrm{G}_{3}\right)$, подвергнутом деформации $u_{33}$.

Эта деформация спаривает $(\mathbf{r}, \mathbf{R})$ узлы иона урана связью 


$$
\Delta H^{\mathrm{cov}}\left[C_{3}\right]=-N_{3} \sum_{\mathbf{r R}} \Gamma_{u}^{\prime}(\rho) u_{33} \tilde{K}_{f d}\left(l_{3} L_{\mathbf{r}}^{3}\right)\left(l_{3} L_{\mathbf{R}}^{3}\right)
$$

с коррелятором

$$
\tilde{K}_{f d}(\rho)=\left\langle D_{\mathbf{r}} \bar{D}_{\mathbf{R}}\right\rangle \cdot
$$

Теперь учтем член Хаббарда

$$
H_{H}=U\left(F_{\mathbf{r}} \bar{F}_{\mathbf{r}}\right)^{2} / 2=U\left[\left(F_{\mathrm{r} L} \bar{F}_{\mathrm{r} L}\right)^{2}+\alpha_{L}^{2}\left(l_{i} L_{\mathbf{r}}^{i}\right)\left(l_{j} L_{\mathbf{r}}^{j}\right)\right] / 2,
$$

где $N_{3}-$ число элементов $\Gamma \Gamma-\mathrm{G}_{3}$.

Для $i=j$ (диагональное $u_{j j}$ ) член Хаббарда

$$
\Delta H_{H}\left[\mathbf{L}_{\mathbf{r}}\right]=\alpha_{L}^{2} U L_{i}^{4} / 2 .
$$

Варьируем сумму выражений (2.11) и (2.8), затем также ТДП, находим средний момент

$$
L_{3}^{2}=\left(N_{3} \Gamma_{u}^{\prime} u_{33} K_{f d}\right) /\left(\alpha_{L}^{2} U\right)
$$

и параметр коллективизации пар $\left(L_{\mathrm{r}}^{i} L_{\mathrm{R}}^{i}\right)$

$$
P_{3}(T)=\left\langle L_{3}^{2}\right\rangle=\left(N_{3} \Gamma_{u}^{\prime} u_{33} /\left(\alpha_{L}^{2} U\right)\right) K_{f d}(T),
$$

где

$$
\begin{aligned}
& K_{f d}(T)=\left\langle D_{0} \bar{D}_{0}\right\rangle+\sum_{\mathbf{k}} N_{\mathbf{k}} P_{\mathbf{k}}, \\
& \left\langle D_{0} \bar{D}_{0}\right\rangle=1-\sum_{\mathbf{k}} N_{\mathbf{k}}, P_{\mathbf{k}}=\Gamma^{\prime}(\mathbf{k}) / \Gamma^{\prime}(0) .
\end{aligned}
$$

Плотность ФХС в Бозе-пространстве Фока

$$
N_{\mathbf{k}}=\left\langle D_{\mathbf{k}} \bar{D}_{\mathbf{k}}\right\rangle=\left(\exp \beta E_{\mathbf{k}}-1\right)^{-1}, \quad E_{\mathbf{k}} \cong \Gamma \mathbf{k}^{2},\left\langle D_{0} \bar{D}_{0}\right\rangle=\rho_{D}^{2}(T) .
$$

Ищем коррелятор

$$
K_{d f}(T)=1-\sum_{\mathbf{k}} N_{\mathbf{k}}\left[1-\Gamma^{\prime}(\mathbf{k}) / \Gamma^{\prime}(0)\right],
$$

где

$$
\Gamma^{\prime}(\mathbf{k}) \cong \Gamma^{\prime}(0)\left(1-\omega_{\Gamma} \mathbf{k}^{2}\right),
$$

и

$$
\omega_{\vec{k}}=\partial^{2} \Gamma^{\prime}(\mathbf{k}) / \partial k^{2} \cong \omega_{\Gamma}=\text { const. }
$$


Интеграл

$$
q_{T} T^{m}=\sum_{\mathbf{k}} \omega_{\Gamma} \mathbf{k}^{2} N_{\mathbf{k}} \cong \sum_{\mathbf{k}} \omega_{\Gamma}\left(k_{B} T / \Gamma\right)^{3 / 2} \mathbf{k}^{2}
$$

Это дает

$$
P_{3}(T)=P_{03}\left(1-q_{T} T^{m}\right), \quad P_{03} \cong N_{3} \Gamma_{u}^{\prime} u_{33} /\left(\alpha_{L}^{2} U\right),
$$

где

$$
m=3 / 2, \quad q_{T}=\left(k_{B} / \Gamma\right)^{3 / 2} .
$$

Подставляем (2.19) в (2.13) и (2.8) и усредняем пары МЭОС. Получаем ТДП «орбитального стекла» (порядка ГГ- $\mathrm{G}_{3}$ ) в форме

$$
\Delta \Phi=Q_{L} \sum_{i} N_{i} P_{i}^{4}(T),
$$

что подтверждает топологический процесс упорядочения «орбитального стекла». Разрушение этого порядка, согласно (2.13), идет как при нарастании плотности ФХС $\left(N_{k}\right)$, так и при росте объемных деформаций $\omega$, т.е. $u_{j j}=\omega / 3$, что приводит к перемешиванию (ГГ$\mathrm{G}_{3}$ ) и $N_{j}=N / 3$, где $N-$ полное число пар ионов урана.

\section{3. «ОРБИТАЛЬНОЕ СТЕКЛО» В МАГНИТНОМ ПОЛЕ В, ВОСПРИИМЧИВОСТЬ МЕТАЛЛИЧЕСКОГО УРАНА (TEH3OP $\chi$ )}

Группы $\left(\Gamma \Gamma-\mathrm{G}_{3}\right)$ [5-7] разделяют кубическое пространство металлического урана на зоны $C_{j}(j=1-3$ или $x, y, z)$. Выделяется параметр порядка $P_{3}$, и группа $\left(\Gamma \Gamma-\mathrm{G}_{3}\right)$ вводит

$$
H_{B}^{i}[\mathbf{L}, \mathbf{r}]=-\sum_{\mathbf{r}} \mathbf{B} \mathbf{L} \mu_{j}-\sum_{\mathbf{r R}} \Gamma_{j u}^{\prime} L_{\mathbf{r}}^{j} L_{\mathbf{R}}^{j}+U_{F} \sum_{\mathbf{r}}\left(L_{\mathbf{r}}^{j}\right)^{4} / 2 .
$$

Вариация ( $\mu_{j}-$ «орбитальный магнетон Бора», будет определен в другом месте)

$$
\frac{\delta H_{B}^{j}}{\delta L_{\mathbf{r}}^{j}}=-B_{j} \mu_{j}+2 U_{F}\left(L_{\mathbf{r}}^{j}\right)^{3}-\sum_{\vec{\rho}} \Gamma_{j u}^{\prime}(\rho) L_{\mathbf{R}}^{j}
$$

при заданном пределе $N_{3}$ группы $\left(\Gamma \Gamma-\mathrm{G}_{3}\right)$ и $N_{1}=N_{2}=0$ дает $L_{j}^{2}$.

Из (3.2) получаем обобщение $\left(\Gamma \Gamma-\mathrm{G}_{3}\right)$ в форме

$$
L_{\mathbf{r}}^{j}(\mathbf{B})=L_{\mathbf{r}}^{j}(0)+\Delta L_{\mathbf{r}}^{j}(\mathbf{B}),\left[L_{\mathbf{r}}^{j}(0)\right]^{2}=P_{3}=\sum \Gamma_{j u}^{\prime} K(T) / U_{F} .
$$

Ее первая вариация 


$$
\Delta L_{\mathbf{r}}^{j}(\mathbf{B})=B_{j} \mu_{j}\left[\frac{\tilde{\Gamma}_{j u}^{\prime} K(T)}{U_{F}}\right]^{-1 / 2}
$$

дает магнитный момент металлического урана

$$
M_{3}=\mu_{3}\left\langle\Delta L_{\mathbf{r}}^{3}\right\rangle=B_{3} \mu_{3}^{2} N_{3} P_{3}(T)
$$

и магнитную восприимчивость «орбитального стекла»

$$
\chi_{33}=\partial M_{3} / \partial B_{3}=\mu_{3}^{2} N_{3} P_{3}(T)>0 .
$$

Этот парамагнетизм металлического урана достаточен для его экспериментальной проверки. Он определяет как предел $N_{3}$ группы $\left(\Gamma \Gamma-\mathrm{G}_{3}\right)$, так и ход $P_{3}$ в области существования «орбитального стекла».

\section{4. МАГНИТОУПРУГОСТЬ «ОРБИТАЛЬНОГО СТЕКЛА»}

Группы ГГ- $\mathrm{G}_{3}$ выделяют пары $\mathbf{L}_{\mathbf{r}}$ и $\mathbf{L}_{\mathbf{R}}$ соседей $(\mathbf{r}, \mathbf{R})$ зоны $\mathrm{C}_{3}$ (пространства металлического урана). Поскольку направленность $G_{j}$ выделяется зонной деформацией $u_{j j}$, добавляется анизотропный член ковалентной связи

$$
\Delta H_{u}\left[L_{\mathbf{r}}^{j}\right]=-\sum_{\mathbf{r R}} \Gamma_{u}^{\prime} u_{j j} \mathbf{K}_{f d} \alpha_{L}^{2} L_{\mathbf{r}}^{j} L_{\mathbf{R}}^{j} .
$$

Он определяется неоднородностью Г $(\rho)$ связи $(5 f-6 d)$ ионов $(\mathbf{r}, \mathbf{R})$ металлического урана и их $(5 f-6 d)$ коррелятором $K_{f d}$ (см. разд. 3 ). Тогда магнитоупругие константы урана будут следствием (4.1).

Варьируя сумму (4.1) и

$$
\Phi[\mathbf{u}]=\mathbf{C u} \otimes \mathbf{u} / 2, \quad \Phi\left[u_{33}\right]=C_{3} u_{33}^{2} / 2,
$$

получаем магнитострикционную деформацию

$$
u_{j j}(T)=N_{3} \sum_{\vec{k}} \Gamma^{\prime}(\mathbf{k}) K_{f d}(\mathbf{k}) \alpha_{L}^{2} P_{3}(T)
$$

и магнитоупругий параметр

$$
\Lambda_{33}=N_{3} \sum_{\mathbf{k}} \Gamma^{\prime}(\mathbf{k}) K_{f d}(\mathbf{k}) P_{3}(T), P_{3}=\left\langle\left(L_{\mathbf{r}}^{3}\right)^{2}\right\rangle .
$$

Его вид определяется концентрацией ионов $(\mathbf{r}, \mathbf{R})$ пространства $C_{3}$ $\left(\Gamma \Gamma-\mathrm{G}_{3}\right)$, а температурная зависимость - параметром «орбитального стекла» $P_{3}(T)(2.17)$ и коррелятором $K_{f d}(\mathbf{k})$ сложного вида.

Запишем теперь ТДП 


$$
\varphi(T)=\sum_{\mathbf{k}} \Gamma^{\prime}(\mathbf{k}) K_{f d}(\mathbf{k})=\Gamma^{\prime}(0)\left\langle D_{0} \bar{D}_{0}\right\rangle \rho_{F}^{2}+\sum_{\vec{k}} \rho_{F}^{2} \Gamma^{\prime}(\mathbf{k}) N_{\mathbf{k}},
$$

где

$$
\rho_{F}^{2}=\left\langle F_{0} \bar{F}_{0}\right\rangle \cong 1,\left\langle D_{\mathbf{k}} \bar{D}_{\mathbf{k}}\right\rangle=\left\langle N_{\mathbf{k}}\right\rangle \cong k_{B} T / \Gamma^{\prime}(0)
$$

и

$$
\Gamma^{\prime}(\mathbf{k}) \cong \Gamma^{\prime}(0) \mathbf{k}^{2},
$$

откуда магнитоупругий параметр

$$
\Lambda_{33}(T) \cong N_{3} P_{3}(T) \rho_{F}^{2}(0)\left[1-k_{\mathrm{B}} T / \Gamma^{\prime}(0)\right] .
$$

Величины магнитоупругих деформаций $u_{j j}$ можно измерить по изменению параметра решетки урана из-за переориентации $\left(\Gamma \Gamma-\mathrm{G}_{3}\right)$. Ее можно вызвать как внешним упругим напряжением $\sigma_{i j}$, так и, в принципе, приложением большого внешнего магнитного поля В (или электрического E), за счет парамагнитной энергии или (3.2).

Другой путь разрушения «орбитального стекла» - нарушение антипараллельности $\left(\mathbf{L}_{\mathbf{r}} \uparrow \downarrow \mathbf{L}_{\mathbf{R}}\right)$ пар.

\section{5. СПЕКТР ФХС, ВОЗБУЖДЕНИЯ 6d- И 5f-ЗОН ФХС}

Ковалентные связи $6 d(\mathbf{r})-6 d(\mathbf{R})$

$$
H^{d d}=-\sum_{\mathbf{r} \mathbf{R}} \Gamma^{d d}(\mathbf{r}-\mathbf{R}) D_{\mathbf{r}} \bar{D}_{\mathbf{R}}=-N \xi_{d}^{2}\left\{\Gamma^{d d}(0) D_{0} \bar{D}_{0}+\sum_{\mathbf{k}} \Gamma^{d d}(\mathbf{k}) D_{\mathbf{k}} \bar{D}_{\mathbf{k}}\right\}
$$

возбуждают ФХС

$$
E_{\mathbf{k}}=\Gamma(0)-\Gamma(\mathbf{k}) \cong \Gamma_{d d} k^{2}, \quad N_{\mathbf{k}}^{d}=\left(\exp \beta E_{\mathbf{k}}-1\right)^{-1} .
$$

Гибридизация $5 f-6 d$ добавляет ковалентные связи типа

$$
H_{F d}=-\sum_{\mathbf{r R}}\left[\Gamma^{F d}(\mathbf{r}-\mathbf{R}) F_{\mathbf{r}} \bar{D}_{\mathbf{R}}+\text { H.c. }\right]
$$

или

$$
H_{F d}=N \xi_{d} \xi_{2}\left[\Gamma_{F d}(0) D_{0} \bar{F}_{0}+\sum_{\mathbf{k}} \Gamma_{F d}(\mathbf{k}) F_{\mathbf{k}} \bar{D}_{\mathbf{k}}+\text { H.c. }\right] .
$$

Первый член в (5.3') учитываем как часть ТДП стабилизации решеток.

Спектр ФХС ищем из уравнений для функций Грина

$$
G_{\mathrm{k}}^{d}=\left\langle\left\langle\bar{D}_{\mathrm{k}} \mid D_{\mathrm{k}}\right\rangle\right\rangle, G_{\mathrm{k}}^{F}=\left\langle\left\langle\bar{F}_{\mathrm{k}} \mid D_{\mathrm{k}}\right\rangle\right\rangle,
$$


а именно

$$
\left(\begin{array}{cc}
\left(E-E_{\mathbf{k}}\right) & \left(\xi_{2} / \xi_{d}\right) \Gamma_{F d}^{*}(\mathbf{k}) \\
\left(\xi_{d} / \xi_{2}\right) \Gamma_{F d}(\mathbf{k}) & \left(E-b_{\mathbf{k}}\right)
\end{array}\right)\left(\begin{array}{c}
G_{\mathbf{k}}^{q} \\
G_{\mathbf{k}}^{F}
\end{array}\right)=\left(\begin{array}{c}
1 /\left(N \xi_{2}^{2}\right) \\
0
\end{array}\right),
$$

где инфинитезимальный член $b_{\mathrm{k}} \rightarrow 0$.

Детерминант (5.5)

$$
\Delta_{2}=\left(E-E_{\mathbf{k}}\right)\left(E-b_{\mathbf{k}}\right)-\left|\Gamma_{F d}\right|^{2} \rightarrow 0
$$

дает спектр ФХС.

\section{6. ЗОННЫЙ СПЕКТР В ОБЛАСТИ «ОРБИТАЛЬНОГО СТЕКЛА»}

Статистика $\Gamma \Gamma-\mathrm{G}_{3}$ приводит к анизотропии поверхности Ферми эффективной массы зонных фермионов $\left(m_{i j}^{*}\right)$. Важнейшую роль играет взаимодействие зонных фермионов и ФХС. Ковалентно-зонный гамильтониан

$$
H^{b-\operatorname{cov}}=\sum_{\mathbf{r R}} \gamma_{F b}(\rho) F_{\mathbf{r}} f_{\mathbf{R}}^{+} f_{\mathbf{r}} F_{\mathbf{R}}
$$

после разложения орбитального фактора МЭОС

$$
v_{\mathrm{rL}}=\left(1+\alpha_{L} l_{i} L_{\mathrm{r}}^{i}\right)^{1 / 2} \cong 1+\alpha_{L} l^{i} L_{\mathrm{r}}^{i} / 2
$$

выделяет орбитальный член ковалентно-зонной связи

$$
\gamma_{F b}^{j j}\left[\rho, L_{\mathbf{r}}^{j}\right]=\gamma_{F b}(\rho)\left(1+\alpha_{L}^{2} L_{\mathbf{r}}^{j} L_{\mathbf{R}}^{j} / 4\right) .
$$

Используем усреднение (6.3) в рамках $\left(\Gamma \Gamma-\mathrm{G}_{3}\right)$

$$
\left\langle L_{\mathbf{r}}^{j} L_{\mathbf{R}}^{j}\right\rangle=P_{3}(T), \mathbf{L}_{\mathbf{r}} \uparrow \downarrow \mathbf{L}_{\mathbf{R}} .
$$

Теперь параметр «орбитального стекла» $P_{3}$ входит в расчеты зонного спектра $\tilde{\varepsilon}_{k}\left(P_{3}\right)$, на основе (5.1), (5.2).

Разложение МЭОС (6.2) приводит к суммарному гамильтониану с выделением

$$
\left\langle F_{\mathbf{k}} \bar{F}_{\mathbf{k}}\right\rangle=\left\langle D_{\mathbf{k}} \bar{D}_{\mathbf{k}}\right\rangle \cdot \Theta_{F D}, \quad\left(\Gamma_{d d} / \Gamma_{F D}\right)^{p} \sim \Theta_{F D} .
$$

Переходный фактор между плотностями $\Phi \mathrm{XC} \mathrm{в} \mathrm{зонах} 6 d$ и $5 f$

$$
\Theta_{F D} \cong\left(\Gamma_{F D} / \Gamma_{d d}\right) \text { при } p \sim-1,
$$

см. в [1]. Обратимся (ниже) к конкретным результатам в разд. 7. 


\section{7. ЭЛЕКТРОСОПРОТИВЛЕНИЕ ПЕРЕХОДНЫХ МЕТАЛЛОВ И МЕТАЛЛИЧЕСКОГО УРАНА}

При сравнимых температурах Дебая $T_{D} \sim 300-400 \mathrm{~K}$ электросопротивление $R$ непереходных $(\mathrm{Cu}, \mathrm{Au}, \mathrm{Ag})$ и переходных $(\mathrm{Fe}, \mathrm{Co}, \ldots)$ металлов различаются по порядку величины. Полагая, что рассеяние токовых электронов на фононах сравнимо (при сравнимых массах ионов вклады фононного рассеяния в $R(T)$ должны быть сравнимы). Но ковалентные связи неполных $3 d$-оболочек через МЭОС приводят к рассеянию зонных фермионов на ФХС. Их вклад в $R(T)$ значительно превосходит фононный.

Основной $(\Phi \mathrm{XC)}$ вклад в рассеяние зонных фермионов дается зонно-ковалентным гамильтонианом

$$
H^{b-\operatorname{cov}}=\sum_{\mathbf{k}} E_{\mathbf{k}} D_{\mathbf{k}} \bar{D}_{\mathbf{k}}+\sum_{\mathbf{k q q}} \gamma D_{\mathbf{q}^{\prime}} f_{\mathbf{k}}^{+} f_{\mathbf{k}+\mathbf{q}} \bar{D}_{\mathbf{q}}+\sum_{\mathbf{k}} \tilde{\varepsilon}_{\mathbf{k}} f_{\mathbf{k}}^{+} f_{\mathbf{k}} .
$$

Находим его методом функций Грина

$$
G_{\mathrm{k}}^{b}=\left\langle\left\langle f_{\mathbf{k}} \mid f_{\mathrm{k}}^{+}\right\rangle\right\rangle, \quad G_{\mathrm{kqq}}^{b c}=\left\langle\left\langle D_{\mathbf{q}} f_{\mathrm{k}+\mathbf{q}} \bar{D}_{\mathbf{q}^{\prime}} \mid f_{\mathrm{k}}^{+}\right\rangle\right\rangle
$$

и уравнениями для них

$$
\begin{gathered}
\left(E-\tilde{\varepsilon}_{\mathbf{k}}\right) G_{\mathrm{k}}^{b}-\sum_{\mathbf{q} q^{\prime}} \gamma G_{\mathrm{kqq}}^{b c}=1, \\
\left(E-\Gamma_{\mathbf{q}}+\Gamma_{\mathbf{q}^{\prime}}-\tilde{\varepsilon}_{\mathbf{k}+\mathbf{q}}\right) G_{\mathbf{k q q ^ { \prime }}}^{b c}-\sum_{\mathbf{k}^{\prime} \mathbf{p}} \gamma\left\langle\left\langle f_{\mathbf{k}+\mathbf{q}} D_{\mathbf{q}^{\prime}} f_{\mathbf{k}^{\prime}}^{+} f_{\mathbf{k}^{\prime}+\mathbf{p}} \bar{D}_{\mathbf{q}} \mid f_{\mathbf{k}}^{+}\right\rangle\right\rangle,
\end{gathered}
$$

где выделяем члены

$$
\left(\delta_{\mathbf{q}^{\prime} 0} \delta_{\mathbf{k}+\mathbf{p}, \mathbf{k}+\mathbf{q}} \delta_{\mathbf{k}^{\prime}+\mathbf{p}, \mathbf{k}} \delta_{\mathbf{k}+\mathbf{q}, \mathbf{k}^{\prime}}\right) \text { и }\left(\delta_{\mathbf{q}^{\prime} 0} \delta_{\mathbf{p} 0}\right)
$$

которые дадут

$$
\left\langle\left\langle f_{\mathrm{k}^{\prime}}^{+} f_{\mathrm{k}^{\prime}} f_{\mathrm{k}} \mid f_{\mathrm{k}}^{+}\right\rangle\right\rangle\left\langle D_{0} \bar{D}_{0}\right\rangle=\rho_{D}^{2} n_{\mathrm{k}^{\prime}} G_{\mathrm{k}}^{b}, \quad n_{\mathrm{k}}=\left\langle f_{\mathrm{k}}^{+} f_{\mathrm{k}}\right\rangle .
$$

Одна часть решения (7.3)

$$
G_{\mathrm{kqk}}^{b c}=\gamma \bar{n}_{\mathbf{k}^{\prime}} \rho_{D}^{2} /\left(E-\tilde{\varepsilon}_{\mathbf{k}+\mathbf{q}}-\Gamma_{\mathbf{q}}\right), \quad \bar{n}_{\mathbf{k}}=N_{b} / N
$$

и ее перенормировка спектра зонных фермионов

$$
\Delta \varepsilon_{\mathbf{k}}+\sum_{\mathbf{q}}|\gamma|^{2} \bar{n}_{\mathbf{k}^{\prime}} \rho_{D}^{2} /\left(\varepsilon_{\mathbf{k}}-\varepsilon_{\mathbf{k}+\mathbf{q}}-\Gamma_{\mathbf{q}}\right)
$$

дает

$$
\tau_{0}^{-1}=2 \pi|\gamma|^{2} N_{b} \rho_{D}^{2}(T) \sum_{\mathbf{q}} \delta\left(\varepsilon_{\mathbf{k}}-\varepsilon_{\mathbf{k}+\mathbf{q}}-\Gamma_{\mathbf{q}}\right)
$$


для частоты релаксации зонных фермионов.

Этот член играет роль при $T=0$ К, поскольку по формуле Друде

$$
R_{0}(0) \sim \tau_{0}^{-1}=Q(T) J_{0}, \quad Q(T)=\text { const } \cdot \rho^{2}(\mathbf{r}), \quad J_{0} \cong \text { const. }
$$

Полагаем $\rho^{2}(0)=1$ и получаем остаточное электросопротивление переходного металла $(7.8)$ и

$$
R_{T}(T) \sim \tau_{T}^{-1}=2 \pi \sum_{\mathbf{k}} \operatorname{Im} \Delta \varepsilon_{\mathbf{k}}(T),
$$

как интегральное рассеяние зонных фермионов на спектре ФХС.

Здесь вклад в перенормировку спектра зонных фермионов дают (7.5) и (7.6), а из

$$
\left\langle\left\langle f_{\mathbf{k}+\mathbf{q}} D_{\mathbf{q}+\mathbf{k}^{\prime}} f_{\mathbf{k}^{\prime}}^{+} f_{\mathbf{k}^{\prime}+\mathbf{p}} \bar{D}_{\mathbf{q}^{\prime}} \mid f_{\vec{k}}^{+}\right\rangle\right\rangle \cong G_{\mathbf{k}}^{b} \delta_{\mathbf{p} \mathbf{q}^{\prime}} \delta_{\mathbf{k}^{\prime}+\mathbf{p}, \mathbf{k}+\mathbf{q}} n_{\mathbf{k}+\mathbf{q}} N_{\mathbf{q}}
$$

получаем перенормировку параметра связи

$$
\gamma(\mathbf{L})=\gamma(0)+\gamma^{\prime} u_{i j}\left(l_{i} L_{\mathbf{r}}^{i}+l_{j} L_{\mathbf{R}}^{j}\right) .
$$

Учет орбитальных факторов МЭОС сдвигает спектр зонных фермионов

$$
\Delta \varepsilon_{\mathrm{k}}(T)=\sum_{\mathbf{q}} n_{\mathrm{k}+\mathbf{q}} N_{\mathrm{q}}|\gamma|^{2} /\left(E-\tilde{\varepsilon}_{\mathrm{k}+\mathbf{q}}-\Gamma_{\mathrm{q}}\right),
$$

его реальная часть

$$
\operatorname{Re} \Delta \varepsilon_{\vec{k} j}=\gamma^{2}(0)+\left(\gamma^{\prime} u_{j j}\right)^{2} L_{j}^{2} C_{j}, \quad C_{j}=N_{j}\left[\Gamma \Gamma-\mathrm{G}_{3}\right] .
$$

Отсюда эффективная масса зонных фермионов получает вид тензора в области $\left(\mathrm{C}_{3}, \Gamma \Gamma-\mathrm{G}_{3}\right)$

$$
m_{j}^{*}=m_{0}^{*}+\Delta m_{j}^{*}, \quad \Delta m_{j}^{*} \cong\left(\gamma^{\prime} u_{j j}\right)^{2} L_{j}^{2} N_{j}\left[G_{j}\right] .
$$

В других областях $C\left(\Gamma \Gamma-\mathrm{G}_{3}\right)$ добавки $\Delta m_{j j}^{*}=0$, при $N_{j}=0$ из-за отсутствия там «орбитального стекла».

Далее используем (7.13) как влияние топологии $\Gamma \Gamma-\mathrm{G}_{j}$ на тензор электросопротивления $R_{i j}$.

\section{8. ТЕМПЕРАТУРА ПЛАВЛЕНИЯ АКТИНИДОВ $T_{L}(M)$}

При фазовом переходе первого рода (плавлении) сравниваются энергии (ТДП) кристаллического и жидкого состояний. Основы ТДП жидкого состояния суть кинетические энергии ионов $\Phi_{L}$. Их зависимости $\Phi_{L}(M)$ от массы $M$ иона предполагают близкие значе- 
ния $k_{B} T_{L}$ для близких значений $M$ актинидов

$$
k_{B} T_{L}=E_{b}, T_{L}(M)=\text { const } \cong 10^{3} \mathrm{~K},
$$

давая близкие значения энергии зонных фермионов $E_{b}(T)$ вблизи $T_{L}$.

Наличие $6 d$-оболочки $\mathrm{U}$ изменяет правую часть (8.1) добавлением энергии $6 d-6 d$ ковалентной связи

$$
\Phi_{D}=-\left\langle\sum_{\mathbf{r}, \mathbf{R}} \Gamma D_{\mathbf{r}} \bar{D}_{\mathbf{R}}\right\rangle \cong-\Gamma_{p}(0) n_{d}\left\langle D_{0} \bar{D}_{0}\right\rangle \cong-\Gamma_{p}(0) n_{d} .
$$

Число $6 d$-электронов варьирует в ряду актинидов

$$
n_{d}(M)=0(\mathrm{~Np}, \mathrm{Pu}, \mathrm{Am}), \quad n_{d}=1(\mathrm{U}, \mathrm{Cm}, \mathrm{Cf}), n_{d}=2(\mathrm{Th}, \mathrm{Pa}, \mathrm{Bk}) .(8.3)
$$

Соответственно варьируют их $T_{L}(M)$, см. рис. 1 .

При сравнимых массах ионов $M$ реакция на их колебания увеличивается на величину $\sim \Gamma_{p} n_{d}$. Поэтому для $T_{L}(M)$ имеем вместо $(8.1)$

$$
k_{B} T_{L}=E_{b}+n_{d} \Gamma_{p}(0), \text { или } \Delta T_{L} \sim 10^{2} \mathrm{~K} \sim \Gamma_{p}(0) \text { для } \mathrm{U} \text { и } \mathrm{Cm} .
$$

Оценка (8.4) хорошо представляет ход $T_{L}(M)$ рис. 1 , тогда как ход температуры Дебая $T_{D}(M)$ заметно отличается от рис. 1 . Объяснение сего факта будет иллюстрировано расчетом спектра фононов U в

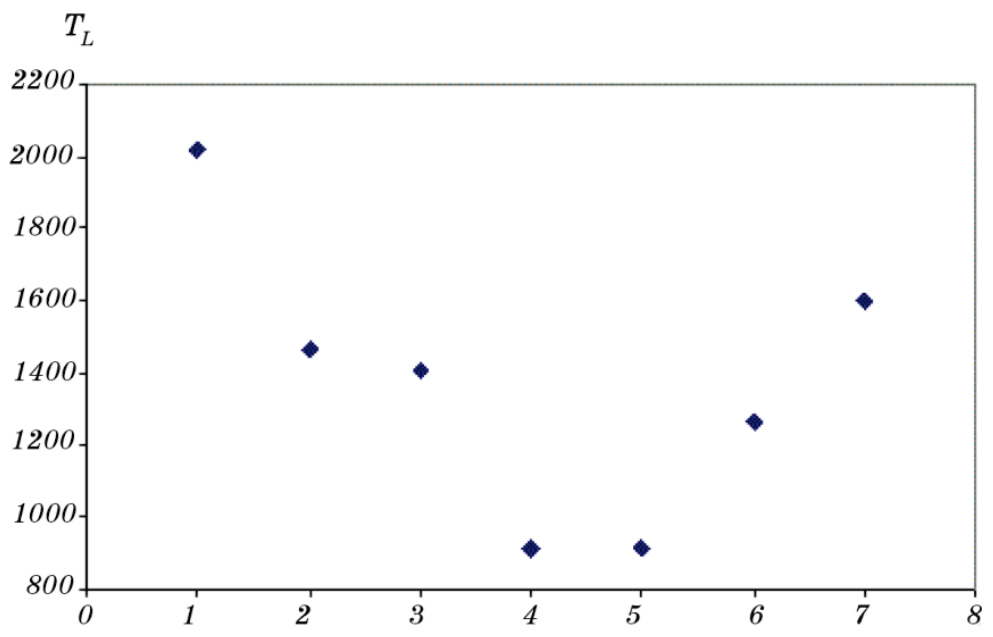

Рис. 1. Температуры плавления актинидов: $1-\mathrm{Th}, 2-\mathrm{Pa}, 3-\mathrm{U}, 4-\mathrm{Np}$, $5-\mathrm{Pu}, 6-\mathrm{Am}, 7-\mathrm{Cm}$.

Fig. 1. Melting temperatures of actinides: $1-\mathrm{Th}, 2-\mathrm{Pa}, 3-\mathrm{U}, 4-\mathrm{Np}, 5-$ $\mathrm{Pu}, 6-\mathrm{Am}, 7-\mathrm{Cm}$. 
другом месте.

Сравнение с классической теорией получаем, рассматривая конфигурационную энтропию порядка «орбитального стекла». Вероятности элементов групп $\left(\Gamma \Gamma-\mathrm{G}_{j}\right)$ выражаются через параметр порядка «стекла», т.е. через $P_{j}(T)$. Этот простой расчет очевиден и здесь не приводится. В частности, из-за чисто квантовой природы элементов групп Галуа, операции перестановки элементов групп (конфигурационная энтропия) выражаются через изменения ковалентных связей.

\section{9. АНОМАЛЬНЫЙ ЭФФЕКТ ХОЛЛА}

Тензор электросопротивления (ЭС) имеет диагональные (ДЭС) и недиагональные (НЭС) компоненты. Носители магнитного порядка $\left(\mathbf{S}_{\mathbf{r}}\right.$ и $\left.\mathbf{L}_{\mathbf{r}}\right)$ обусловливают в НЭС нечетные ферромагнитные явления (аномальный эффект Холла и др.) Компенсация $\left(S_{r}=0\right)$ при $\mathbf{L}_{\mathrm{r}} \neq 0$ должна проявляться эффективно только как следствие неоднородности ковалентной связи

$$
\Gamma_{i j}(\mathbf{r}-\mathbf{R})=\Gamma\left(\rho_{0}\right)+\Gamma_{\rho}^{\prime}\left(\rho_{0}\right) u_{i j}, \quad \Gamma^{\prime}=\partial^{2} \Gamma(\rho) / \partial \rho_{i} \partial \rho_{j} .
$$

Выделяется анизотропная часть ковалентного гамильтониана

$$
H^{d f}=-\sum_{\mathbf{r R}} \Gamma^{d f} F_{\mathbf{r}} D_{\mathbf{R}} \bar{D}_{\mathbf{r}} \bar{F}_{\mathbf{R}}+\Delta H^{d f}
$$

где

$$
\Gamma^{d f}(\rho)=\Gamma^{d f}\left(\rho_{0}\right)+\Gamma_{d f}^{\prime} u_{i j}\left(l_{i} L_{\mathbf{r}}^{i}\right)\left(l_{j} L_{\mathbf{R}}^{j}\right),
$$

что дает

$$
\delta_{i j}\left(L_{\mathbf{r}}^{i} L_{\mathbf{R}}^{i}\right)=\delta_{i j} L^{2} \alpha_{i}^{2}, \quad l_{j}^{2}=\text { const }
$$

для ковалентной энергии.

Получаем гамильтониан

$$
\begin{aligned}
& H=-\sum_{\mathbf{r R}} \Gamma^{f d}(\mathbf{r}-\mathbf{R})+\Gamma u_{i j} \alpha_{i} \alpha_{j} l_{i} L_{\mathbf{r}}^{i} l_{j} L_{\mathbf{R}}^{j}\left(F_{\mathbf{r}} D_{\mathbf{R}} \bar{D}_{\mathbf{r}} \bar{F}_{\mathbf{R}}+\ldots\right)+\sum_{\mathbf{k}} \tilde{\varepsilon}_{\mathbf{k}} f_{\mathbf{k}}^{+} f_{\mathbf{k}}+ \\
& +\sum_{\mathbf{r R}}\left[\gamma_{b d}(\rho) D_{\mathbf{r}} f_{\mathbf{R}}+\text { H.c. }\right]+\sum_{\mathbf{r R}}\left(\gamma_{F b} F_{\mathbf{r}} f_{\mathbf{R}}+\text { H.c. }\right)\left(1+\alpha_{f i} u_{i j} l_{i} L_{\mathbf{r}}^{i}+l_{j} L_{\mathbf{R}}^{j}\right) .
\end{aligned}
$$

Для функций Грина

$$
G_{\mathrm{k}}^{b}=\left\langle\left\langle f_{\mathrm{k}} \mid f_{\mathrm{k}}^{+}\right\rangle\right\rangle, G_{\mathrm{r}}^{d}=\left\langle\left\langle\bar{D}_{\mathrm{k}} \mid f_{\mathrm{k}}^{+}\right\rangle\right\rangle, G_{\mathrm{k}}^{F}=\left\langle\left\langle\bar{F}_{\mathrm{k}} \mid f_{\mathrm{k}}^{+}\right\rangle\right\rangle
$$

получаем добавки к уравнениям 


$$
\left(E-\tilde{\varepsilon}_{\mathbf{k}}\right) G_{\mathbf{k}}^{b}-\gamma_{b F}\left\{1+\alpha_{L} u_{i j}\left(l_{i} L_{\mathbf{r}}^{i}\right)\left(l_{j} L_{\mathbf{R}}^{j}\right)\right\} G_{\mathbf{k}}^{F}=1 .
$$

Чтобы найти $G_{\vec{k}}^{F}$, используем другие функции Грина

$$
G_{\mathrm{k}}^{d}=\left\langle\left\langle\bar{D}_{\mathrm{k}} \mid D_{\mathrm{k}}\right\rangle\right\rangle, G_{\mathrm{k}}^{F}=\left\langle\left\langle F_{0} D_{0} \mid \bar{F}_{\mathrm{k}}\right\rangle\right\rangle,
$$

дающие спектры ФХC

$$
E_{d} \cong \Gamma_{d d} \mathbf{k}^{2}, \quad E_{f} \cong\left[\left|\gamma_{d f}(\mathbf{k})\right|^{2} / \Gamma_{d d}(\mathbf{k})\right]\left[1+u_{i j} \alpha_{i} \alpha_{j}\left\langle L_{i}^{2} L_{j}^{2}\right\rangle\right] .
$$

Воспользуемся преобразованием

$$
\Delta H^{b c}=\sum_{\mathrm{rR}} \gamma_{0}^{\prime} u_{i j}\left(1+l_{i} L_{\mathrm{r}}^{i} / 2\right)\left(1+l_{j} L_{\mathrm{R}}^{2}\right),
$$

согласно

$$
\gamma_{i j}^{\prime}=\gamma_{0}^{\prime}\left(N_{i} L_{i}^{2}+N_{j} L_{j}^{2}\right) \rightarrow \gamma_{0}^{\prime}\left(N_{3} P_{3}+N_{1} P_{1}\right) .
$$

Оно дает тензор релаксации зонных фермионов в анизотропной форме (7.12).

Подставляем его в формулу Друде для тензора электросопротивления. Получаем недиагональный член

$$
R_{31}=R_{0 c}\left(N_{3} P_{3}+N_{1} P_{1}\right)
$$

для описания аномального эффекта Холла в явной форме. В зоне $C_{3}$ «орбитального стекла" константа Холла выражается через числа элементов групп Галуа $N_{3}$ и $N_{1}$, а также через параметры «стекла» $P_{j}$. Анизотропия тензора релаксации зонных фермионов коррелирует с анизотропией эффективной массы зонных фермионов, т.е. поверхности Ферми.

\section{0. ЗАКЛЮЧЕНИЕ И ОБЩИЕ ВЫВОДЫ}

Применение $\mathrm{U}^{238}$ требует тщательного измерения его свойств как в чистом металлическом уране, так и в соединениях. Неоднократные попытки использования их в техническом магнетизме дали малоутешительные результаты [8]. Использование $\mathrm{U}^{238}$ как оружия привело скорее к химическим (отравляющим) действиям, нежели к физическим (фугасным) результатам. Наличие значительного локального орбитального момента $L_{\mathrm{r}} \sim 1$ не удается прямо использовать в обстоятельствах зануления спина $\left(\mathbf{S}_{\mathbf{r}}=0\right)$ иона урана. Детальные аспекты использования $\mathrm{U}^{238}$ требуют квантового подхода. Здесь адекватно представление МЭОС [1]. Следует, однако, отметить не укладывающийся в простую схему случай Am. 


\section{1. КОНКРЕТНЫЕ ВЫВОДЫ}

1.Макроупорядочение $\mathbf{L}_{\mathbf{r}}$ (иона урана в узлах $\mathbf{r}$ ) описывается группами Галуа ГГ ориентации $\mathrm{G}_{3}$ (пространство $C_{3}$, элементов группы $\left.N_{3}\right)$. Это «орбитальное стекло» $\left(\mathbf{L}_{\mathbf{r}} \uparrow \downarrow \mathbf{L}_{\mathbf{R}}\right.$ пары ионов урана как элементов ГГ) создается асимметрией деформации $u_{i j}$.

2 . Включение $u_{33}$ зоны $C_{3}$ сводит пары ионов урана $(\mathbf{r}, \mathbf{R})$ в группу $\Gamma \Gamma-\mathrm{G}_{3}$.

3. Параметр порядка «орбитального стекла» $P_{3}=\left\langle\left(L_{3}\right)^{2}\right\rangle$ разрушается флуктуациями химических связей (ФХC) при росте температуры $T$.

4. Топология металлического урана (набор $\Gamma \Gamma-\mathrm{G}_{3}$ ) выражает его свойства через $P_{j}(T)$.

5 . Добавочная часть магнитной восприимчивости $\chi_{j j} \sim P_{j}(T)$ определяется топологией металлического урана.

6. Топология металлического урана прямо связана с тензором $u_{i j}$. Это разделение металлического урана на пространства $C_{j}\left(\Gamma \Gamma-\mathrm{G}_{j}\right)$ создает анизотропию поверхности Ферми зонных фермионов.

7. «Орбитальное стекло» проявляется в магнетосопротивлении и в эффекте Холла через $P_{j}(T)$.

8. Добавочное электросопротивление $R(T)$ за счет рассеяния зонных фермионов на $\Phi Х С$ сильно увеличивает его в переходных металлах и дает остаточное электросопротивление $R_{0}(0)$.

9 . Топология «орбитального стекла» создает анизотропию $R_{i j}(T)$ изза конкуренции разных групп $\left(\Gamma \Gamma-\mathrm{G}_{j}\right)$.

10. Аномальный эффект Холла явно выражается через параметры $P_{3}$ и $P_{1}$ в форме $R_{31}$ и соответствующую константу Холла.

11. Ковалентные $6 d-6 d$-связи увеличивают температуру плавления $T_{L} \cong 10^{3} \kappa$ на $10^{2} \kappa$ для $\mathrm{U}$ и других актинидов, имеющих $6 d$-электроны.

\section{ЦИТИРОВАННАЯ ЛИТЕРАТУРА}

1. А. И. Мицек, В. Н. Пушкарь, Металлофиз. новейшие технол., 41, № 3: 279 (2019).

2. А. И. Мицек, В. Н. Пушкарь, Металлофиз. новейшие технол., 37, № 4: 433 (2015).

3. С. В. Вонсовский, Магнетизл (Москва: Наука: 1971).

4. Ю. П. Ирхин, В. Ю. Ирхин, Электронное строение и физические свойства переходных металлов (Свердловск: Уральский государственный университет: 1989).

5. И. Б. Листинг, Предварительные исследования по топологии (Москва: Гос. технико-теор. издат.: 1932).

6. Н. Бурбаки, Общая топология (Москва: Гос. издат. физ.-мат. лит.: 1959).

7. Н. Чеботарев, Основы теории Галуа (Москва: Гос. технико-теор. издат.: 1934). 
8. А. В. Дерягин, А. В. Андреев, ЖЭТФ, 71, № 9: 1166 (1976).

\section{REFERENCES}

1. O. I. Mitsek and V. M. Pushkar, Metallofiz. Noveishie Tekhnol., 41, No. 3: 279 (2019) (in Russian).

2. O. I. Mitsek and V. M. Pushkar, Metallofiz. Noveishie Tekhnol., 37, No. 4: 433 (2015) (in Russian).

3. S. V.Vonsovskiy, Magnetism (Moscow: Nauka: 1971) (in Russian).

4. Yu. P. Irkhin and V. Yu.Irkhin, Elektronnoe Stroenie i Fizicheskie Svoystva Perekhodnykh Metallov [Electron Structure and Physical Properties of Transition Metals) (Sverdlovsk: Ural State University: 1989) (in Russian).

5. J. B. Listing, Predvaritelnye Issledovaniya po Topologii [Preliminary Research on Topology] (Moscow: Gos. Tekniko-Teor. Izdat.: 1932) (in Russian).

6. N. Bourbaki, Obshchaya Topologiya [General Topology] (Moscow: Gos. Izdat. Fiz.-Mat. Lit.: 1959) (in Russian).

7. N. Chebotarev, Osnovy Teorii Galua [The Principles of Galois Theory] (Moscow: Gos. Tekniko-Teor. Izdat.: 1934) (in Russian).

8. A. V. Deryagin and A. V. Andreev, J. Experimental and Theor. Phys., 71, No. 9: 1166 (1976) (in Russian). 